\title{
Analysis of the Stick-Mode Duration of Dry-Friction Oscillators with a Stochastic Approach
}

\author{
Roberta Lima, Rubens Sampaio, \\ Department of Mechanical Engineering, PUC-Rio, \\ Rua Marquês de São Vicente, 225; 38097, Rio de Janeiro, RJ \\ E-mail: roberta_10_lima@hotmail.com,rrsampaio@puc-rio.br
}

\begin{abstract}
This paper analyzes the behavior of a stick-slip dry-friction oscillator considering the existence of uncertainties in a friction parameter. The system studied is composed by a block connected to a fixed support by a spring. The block moves over a continuous belt that is driven by rollers. The frictional force between the block and the belt is modeled as a Coulomb friction. Solutions to the phase diagram of the case of belt with constant velocity are analytically developed. By numerical simulations, this simpler case is compared with different imposed non-smooth belt velocity configurations, from a deterministic and from a stochastic viewpoint. The focus of the paper is to determine the duration of the stick-and slip-mode parts of the trajectory through the phase diagram. In the stochastic analysis, the belt speed is modeled as a random process. Monte Carlo simulations are employed to compute the statistics of the response of the stochastic dry-friction oscillator. The variable of interest is the duration of the stick-mode of the trajectory.
\end{abstract}

Key words: Stick-slip, friction-induced vibration, nonlinear-dynamics, stochastic modeling.

\section{Introduction}

The nature of dynamic friction forces developed between bodies in contact is extremely complex and affected by many factors: the constitution of the interface, inertia and thermal effects, roughness of the contacting surfaces, history of loadings, the presence of lubricants, and many others $[8,7,3,1]$.

Beside this, the stick-slip vibrations are self-sustained oscillations induced by dry friction and since the friction can be characterized in two qualitatively different parts (kinetic and static frictions)with a non-smooth transition, the resulting motion also has a non-smooth behavior. Thus, stick-slip systems belong to the class of non-smooth systems, such as systems with stops, impacts and hysteresis $[9,2]$.

The absence of a universally accepted friction model and the friction coefficient variability (due to the many factors that can affect it), make the stochastic approach the ideal way to deal with friction models. Random exists on the real friction system because the friction coefficient possesses random behavior [4].

The main motivation of this paper is to better understand the stick-slip behavior on drilling, that is very complicated and appears during $50 \%$ of drilling time. One of the consequences of the stick mode is that, since a constant speed is imposed at the top of the drillstring and during the stick the bit at the bottom does not move, the drillstring is twisted and acts as a spring. In this paper a simpler model of this process is studied. The model is the bare minimum to study stick-slip. It has a block, modeled as a particle, with mass but no dimension; a spring to give an elastic force; and a belt to drive the system. Between the block and the belt there is dry friction. Of course the results are dependent of the dry friction model used. The dynamics of this simple system can be divided in two parts, a slip- and a stick-mode. We call stick when the relative velocity of the block-belt is null in a time-interval, not just an instant. If the relative velocity is non-zero, or zero in isolated points, we call slip. The equation of motion has different roles during stick and slip. Indeed, in the stick-mode, the block moves with the same velocity as the belt and the friction force stays within its bounds; the friction force is not known but the acceleration of the block is known as well as its position. The dynamical equation acts as a constraint to find the 
friction force. In the slip part of the motion one has a standard dynamical problem, solved by integration of the dynamical equation. In the phase plane, while the slip region has non-zero measure, the stick region has zero measure and is a manifold of lower dimension, one or zero.

In this paper different imposed belt velocity configurations are analyzed, from a deterministic and also from an stochastic viewpoint. In the simpler model the belt has constant velocity, but if the belt velocity is not constant the manifold of possibilities for the stick-slip distribution is very rich as, one presumes, in the drilling process. Also, since it is well known that the friction properties of contacting surfaces vary a lot with ambient conditions, as temperature, humidity, state or lubrication, etc, so stochastic modeling is also done. The belt velocity is taken as uncertain and modeled as a random process.

This paper is organized as follows. Section 2 describes a most simple stick-slip oscillator. Section 3 presents the results of the deterministic simulations. The probability model to the uncertain friction force are construct in Section 4and the results of the simulations of the stochastic system are presented in Section 5. Section 6 presents some conclusions.

\section{Deterministic Model of the Stick-Slip Oscillator}

The system analyzed in this paper is composed by a block connected to a fixed support by a spring. The block moves over a continuous belt that is driven by rollers, as shown in Fig. 1.

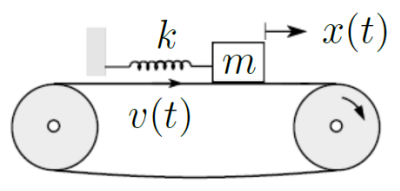

Figure 1: Stick-slip oscillator

The position of the block over the belt is represented by $x$ and its equation of motion, during the slip mode, is

$$
m \ddot{x}(t)+k x(t)=f(t),
$$

where $m$ is the mass of the block, $k$ is the spring stiffness and $f$ is the frictional force between the block and the belt. The belt speed is represented by $v$ and it is assumed that $f$ during the slip mode depends on the slip velocity, $v-\dot{x}$, as

$$
f(t)=n \mu \operatorname{sgn}(v(t)-\dot{x}(t)),
$$

where $n$ is the normal force exercised by the belt on the block and $\mu$ is the friction coefficient. In a simple friction model, this coefficient is assumed to be constant. Next is presented the analysis of the solution of a simple problem, in it the belt velocity is imposed and has a constant speed. To better understand what is stick-slip, the analytical results of the simple stick-slip oscillator with a belt with a constant speed will be later compared to the numerical results of the stick-slip oscillator in the case of imposed non-constant velocity. Considering that the belt speed and $\mu$ are constant in time, and introducing a new variable $y=\dot{x}$, it is possible to write two solutions for the phase paths of the system. Writing $z=x \omega_{n}$, where $\omega_{n}=\sqrt{k / m}$ is the natural frequency of the system, the phase paths become

$$
\text { when } y>v \quad y^{2}+\left(z+\frac{n \mu}{m \omega_{n}}\right)^{2}=c, \quad \text { when } y<v \quad y^{2}+\left(z-\frac{n \mu}{m \omega_{n}}\right)^{2}=c .
$$

where $c$ is a constant. Thus, given a positive $v$, the phase diagram of the system, has a single equilibrium point at $\left(n \mu / m \omega_{n}, 0\right)$. Given a negative $v$, its single equilibrium point is at $\left(-n \mu / m \omega_{n}, 0\right)$, as shown in Fig. 2(a) and 2(b). In both cases, it is a centre [5].

The horizontal segment $y=v$ and $|x| \leq \mu n / k$ correspond to the stick phenomena, i.e., when the block moves with the belt velocity and the friction force is able to compensate the elastic force to maintain the 


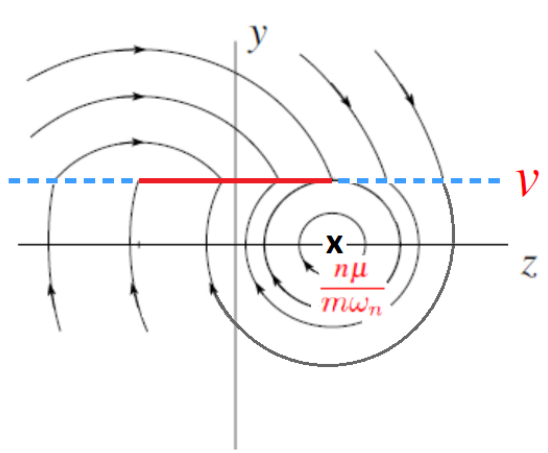

(a)

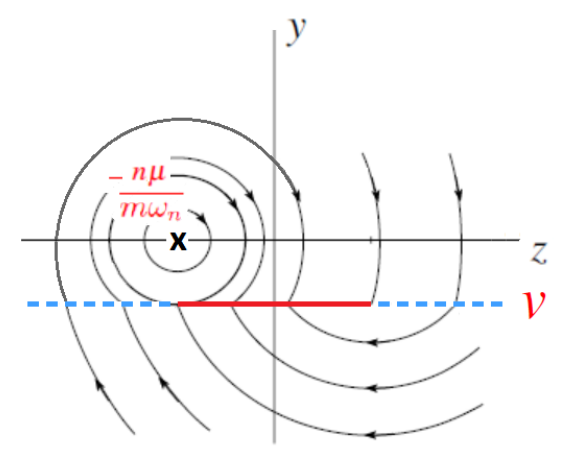

(b)

Figure 2: Phase diagram for the stick-slip oscillator with (a) positive and (b) negative belt speed.

block put. This phenomena can happens only before the system reach the steady-state, because after it, the phase paths are written as a circle with origin in the equilibrium point, Eq. 3, and with radius related with the block initial conditions of the movement.

Thus, if we draw the phase diagram of a stick-slip oscillator with a constant belt speed and Coulomb friction, considering only the steady state solution of the system, there is no stick mode. The path will be written as a circle and it has only slip mode. In the case of circle with radius equal to $|v|$, the path of the block will be tangent to the horizontal segment that corresponds to the stick phenomena. That is, the block and the belt will have in the steady state solution the same velocity in an instant, but this is not what it is considered as stick-mode.

In [6], a simple dry-friction oscillator with Coulomb friction and with different belt velocity configurations was analyzed from a deterministic and from a stochastic viewpoint. It was verified numerically that for non-constant belt speed, it is possible that occur the stick-mode in the steady state solution of the system. But in [6], no results were presented in relation to the duration of the stick-mode.

This paper investigates the same simple dry-friction oscillator of [6] with different belt velocity configurations, but here the objective of is to determine the percent of time in which occur the stickmode during one period of the steady state solution.

\section{Numerical Simulations of the Deterministic Stick-Slip Oscillator}

To better comprehend the behavior of the stick-slip oscillator when the belt do not have a constant speed, simulations with a different model of non-constant belt speed are compared with the analytical results (developed for the case in which the belt has a constant speed) in order to observe their influence in the system response, as in the duration of the stick- and slip-mode.

In all deterministic and stochastic simulations, Eq. (1) was integrated in a range of $[0.0,200.0]$ seconds. For the integration, it was used the function ode45(based on the Runge-Kutta 4th/5th-order method with a varying time-step algorithm) of the Matlab software with a maximal steep size equal to $10^{-4}$ seconds. Also, the maximal absolute error allowed in the integrations was $10^{-4}$.

The values of the parameters used were $1.0[\mathrm{Kg}]$ for the block mass, $4.0[\mathrm{Kg}]$ for the spring stiffness, $1.0[\mathrm{~N}]$ for the normal force, 1.0 for the constant friction coefficient and $v_{0}=1.0[\mathrm{~m} / \mathrm{s}]$ for the modulus of the belt speed. As initial conditions to the system, it was considered $x(0)=0.0[\mathrm{~m}]$ and $\dot{x}(0)=1.0$ $[\mathrm{m} / \mathrm{s}]$. The non-constant belt velocity analyzed is

$$
v(t)= \begin{cases}-v_{0}, & \text { if } t \in\left[0,1 / 2 \omega_{b}\right) ; \\ v_{0}, & \text { if } t \in\left[1 / 2 \omega_{b}, 1 / \omega_{b}\right) .\end{cases}
$$

where $\omega_{b}$ is the frequency of the belt speed. The system behavior with this imposed discontinuous belt speed (a sort of bang-bang control) is affected by the ratio $r=\omega_{b} / \omega_{n}$. 
To understand this influence, we start recalling the analytical form of the phase diagram when the belt has a constant speed, $v$. If the initial condition, $|\dot{x}(0)|>|v|$, the system will have a non steady state solution (in which occurs the stick-mode) and after it, it will have a steady state solution (in which occurs the slip-mode). If $|\dot{x}(0)| \leq|v|$, the system will have only a steady state solution. As it was explained in section 2 , in the steady state solution, the block path is a circle with origin in the equilibrium point, Eq. 3 , and with radius related with the block initial conditions.

Now, we return to the case of belt with discontinuous speed. Each change of the speed sign can be understood as a reboot of the system. Thus, the block position and velocity in the instant that there is change of the speed sign behave as initial conditions to the next time-interval (with size $1 / 2 \omega_{b}$ ) in which the belt speed is constant. The parameter $2 \omega_{b}$ determines the frequency in which the system is rebooted.

The case of small ratio $r$, that is, $\omega_{b}$ is relatively small when compared with $\omega_{n}$, is illustrated in Fig. 3(a) and 3(b). In these figures, the block path when the belt speed is positive is drawn in gray and when is negative is drawn in black. It is possible to observe that, in both graphs, the block path is composed by circles with center at the equilibrium points $\left(n \mu / m \omega_{n}, 0\right)$ and $\left(-n \mu / m \omega_{n}, 0\right)$ (they are marked with a cross). For the ratio $r=0.1$ there is only one circle around each equilibrium point. For the ratio $r=0.4$ is different, there are two circles around each equilibrium point. Also, it is possible to observe that there is no stick-mode during the steady-state of the system.

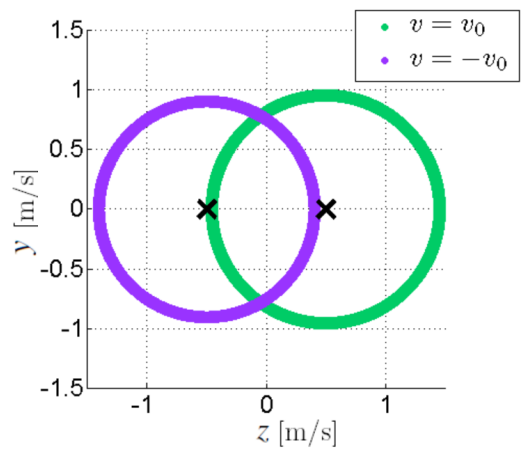

(a)

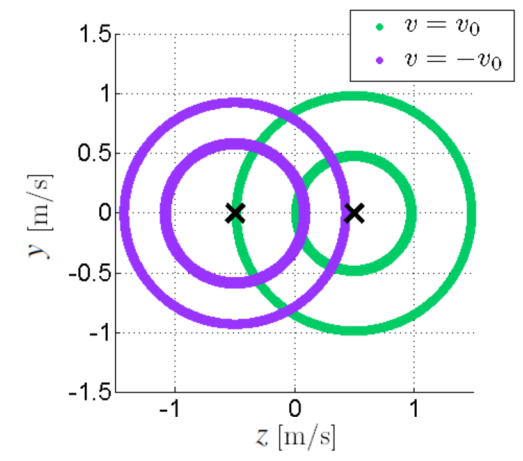

(b)

Figure 3: Belt with discontinuous speed: phase diagram for the stick-slip oscillator in the steady-state solution with (a) $r=0.1$ and (b) $r=0.4$.

When the ratio $r$ is bigger, the phase diagram of the system is not anymore composed by complete circles with center at the equilibrium points $\left(n \mu / m \omega_{n}, 0\right)$ and $\left(-n \mu / m \omega_{n}, 0\right)$, as shown in Fig. 4(a), 4(b), 5(a) and 5(b).

In the case of ratio $r=1.0$ and $r=1.3$, by the numerical results, it was verified that it can occurs the stick and slip modes in the steady state solution of the system. Thus, the phase diagram is composed by arcs of the circles with origin in $\left(n \mu / m \omega_{n}, 0\right)$ and $\left(-n \mu / m \omega_{n}, 0\right)$ (corresponding to the slip-mode) and by horizontal straight segments (corresponding to the stick-mode). In these graphs, the positions considered as stick mode, are drawn with a dashed line. By them, it is possible to verify that, differently from the case in which the belt has a constant speed, the stick mode can occur in the steady state of the system.

In the case of ratio $r=2.0$ and $r=2.5$, it was verified that there is only slip-mode in the steady-state solution of the system. Thus, the phase diagram is composed only by arcs of the circles with origin in $\left(n \mu / m \omega_{n}, 0\right)$ and $\left(-n \mu / m \omega_{n}, 0\right)$.

To better comprehend the behavior of the stick-mode, we will observe the percent of time in which occurs the stick-mode during one period of the steady-state solution of the system. This variable is represented by $t_{s}$, and it was computed for different values of $r$, as shown in Fig. 6 . It is possible to observe that the longer stick-mode occurs when $r$ is around 1.2. In this situation, the duration of the stick-mode is almost $40 \%$ of the period of the steady-state solution of the system. Beside this, it can be seen that for values of $r$ bigger than 1.4, the system do not have any more the stick-mode. 


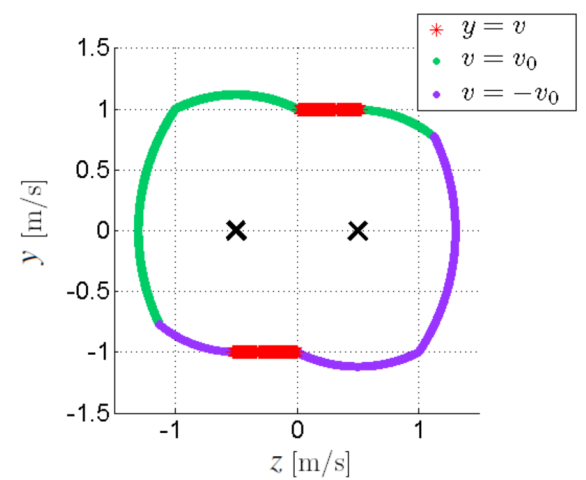

(a)

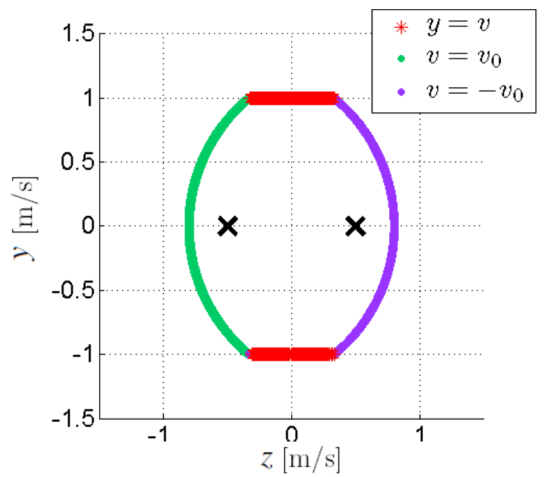

(b)

Figure 4: Belt with discontinuous speed: phase diagram for the stick-slip oscillator in the steady-state solution with (a) $r=1.0$ and (b) $r=1.3$.

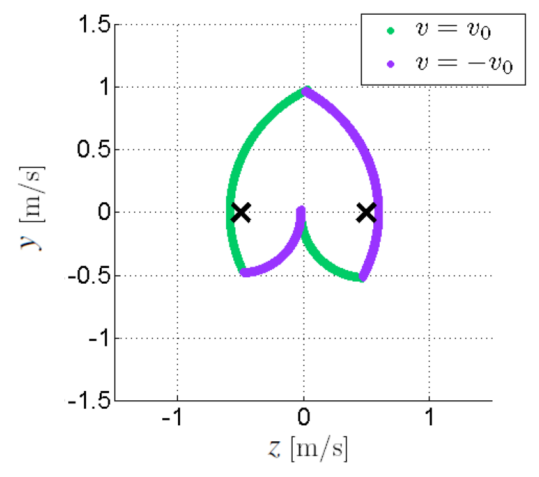

(a)

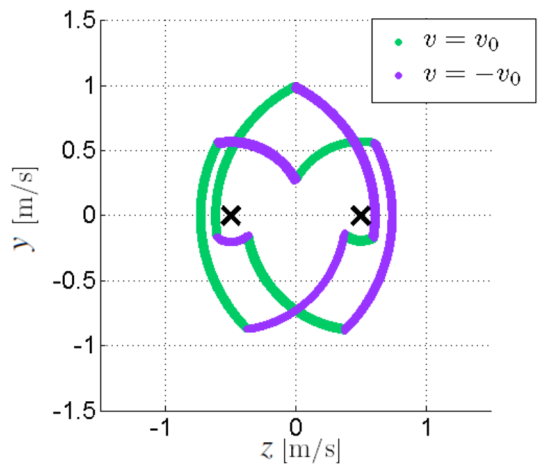

(b)

Figure 5: Belt with discontinuous speed: phase diagram for the stick-slip oscillator in the steady-state solution with (a) $r=2.0$ and (b) $r=2.5$.

\section{Stochastic Model of the Belt Speed}

As it was explained in the introduction, to construct a model of the frictional force is not a simple task. To deal with the friction coefficient variability and the friction dependency on the relative velocity of the bodies in contact, we propose to analyze the stick-slip oscillator with a stochastic approach.

Considering that the belt speed is the a source of uncertainty in the stick-slip oscillator problem, we propose to treat it as a random process with parameter $t$ constant by parts, represented by $\mathcal{V}$. Thus, we define $\mathcal{V}$ as a finite collection of real-valued random variables $\left\{V_{1}, V_{2}, \ldots, V_{n}, \ldots\right\}, \forall n \in \mathbb{N}$, from a probability space $(\Omega, \mathcal{F}, P)$, where $\Omega$ is the sample space, $\mathcal{F}$ is the $\sigma$-algebra and $P$ is the probability measure.

As it is assumed that $\mathcal{V}$ is random process constant by parts, given a time-interval with size $1 / \omega_{b}$,

$$
\mathcal{V}(t)=V_{n} \quad \text { if } \quad t \in\left[\frac{n-1}{\omega_{b}}, \frac{n}{\omega_{b}}\right], \forall n \in \mathbb{N} .
$$

Beside this, it is assumed that $\left\{V_{1}, V_{2}, \ldots, V_{n}, \ldots\right\}$ is a collection of equal and discrete random variables, in which each $V_{n}$ can have only the values $-v_{0}$ and $v_{0}$ with equal probability. 


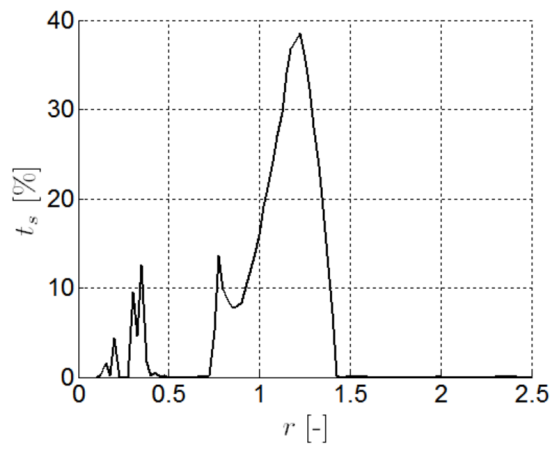

Figure 6: Belt with discontinuous speed: percent of time in which occurs the stick-mode during one period of the steady-state solution of the system.

\section{Numerical Sumulations of the Stick-Slip Oscillator with Random Belt Speed}

As it was assumed that the belt speed is uncertain, modeled as a random process constant by parts, $\mathcal{V}$, the response of the stochastic stick-slip oscillator is a random process with parameter $t$ and thus, the equation of motion of the system, Eq. 1, became a stochastic differential equation. The block displacement is represented by the random process $\mathcal{X}$ and its velocity by $\dot{X}$.

To make the stochastic analysis of the system, Monte Carlo simulations were employed to compute a statistic of the response $X$ and $\dot{X}$ and for each realization of the system, it was found the stick- and slipmode parts of the trajectory. The random variable $T_{s}$ indicates the percent of time in which occurs the stick-mode. As the belt speed is modeled as a random process, each realization of the system response do not have a steady-state behavior, unlike what happened in the deterministic simulations. Therefore, the percentage of time of stick is calculated relative to the total simulation time and no more relative to one period of the steady-state solution. For each realization of the system, one realization of $T_{s}$ is computed adding the duration of all stick-modes and dividing it by the total simulation time. Statistics of this random variable, as mean $\left(\mu_{T_{s}}\right)$ and $90 \%$ confidence interval, were computed for different values of $r$, as shown in Fig. 7. It is possible observe that for values of $r$ between 0.5 and $0.6, \mu_{T_{s}}$ has a linear growth, and after it, reaches a stable level. Comparing this graph with the Fig. 6, it is verified that the deterministic and stochastic systems have a different behavior in relation to the stick-mode duration.

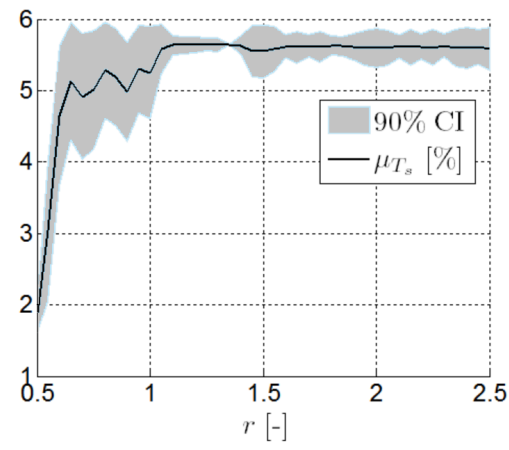

Figure 7: Random belt speed: mean and 90\% confidence interval of $T_{s}$ for different values of $r$.

\section{Conclusions}

The purpose of this paper was to better understand stick-slip oscillators hoping that the better knowledge would help to optimize the drilling process. The focus was in the steady-state, when one was found 
because it could be reproduced, and this way one could be assured of the results. Except in some cases the Coulomb's model for friction was used in the simulations. Deterministic and stochastic situations were analyzed and the focus was in finding the stick- and slip-mode parts of a trajectory. The driving force, the belt motion, was imposed in different ways, in time and space. That is, the velocity of the belt was varied in time and its configuration was uniform and also piecewise uniform. The friction between block-belt is very complex and can only be coarsely described. However, the Coulomb's friction model is surprisingly good in describing the non-smooth dynamics of a stick-slip oscillator. Maybe the mechanism is not the same, but the dynamics of a simple stick-slip oscillator mimics that found in the drilling process.

In the deterministic analysis of the stick-slip oscillator with a constant belt speed and the same surface conditions along its length, it is verified that considering only the steady state solution, there is no stick mode in, when the Coulomb's model is used. The block oscillates around an equilibrium point related with the normal force exerted on the block, the friction coefficient and the natural frequency of system.

When the belt has a different imposed periodic speed, as a discontinuous speed, it is verified, by numerical simulations, that the stick-mode can occur in the steady state of the system, depending on the ratio $r$ between the frequency of the belt speed and the natural frequency of the system.

In the stochastic analysis, assuming that the randomness is in the belt velocity, modeling it as a random process piecewise uniform (characterized by the time-interval $1 / \omega_{b}$ ) and considering zero initial conditions to the block, it was also verified, by numerical simulations, that the stick-mode can occur, depending on the ratio $r$. The percent of time in which occurs the stick-mode is represented by the random variable $T_{s}$ and statistics of it were computed for different values of $r$. Comparing them with the deterministic results, it is observed that the stochastic and deterministic systems have a different behavior in relation to the stick-mode duration.

\section{Acknowledgements}

This work was supported by the Brazilian Agencies CNPQ, CAPES and FAPERJ.

\section{References}

[1] E. Berger, Friction modeling for dynamic system simulation, Appl Mech Rev, vol. 52(6), 2002.

[2] G. Csernák and G. Stépán, On the periodic response of a harmonically excited dry friction oscillator, Journal of Sound and Vibration, vol. 295, pp 649-658, 2006.

[3] B. Feeny, A. Guran, N. Hinrichs and K. Popp, A historical review on dry friction and stick-slip phenomena. Appl Mech Rev, vol. 51(5), 1998.

[4] Q. Feng, A discrete model of a stochastic friction system. Comput. Methods Appl. Mech. Engrg., vol. 192, pp 2339-2354, 2003.

[5] D.W. Jordan and P. Smith, "Nonlinear ordinary differential equations: an introduction for scientists and engineers". Oxford University Press, fourth edition, 2007.

[6] R. Lima and R. Sampaio, Some Remarks About Stick-Slip Oscillators, Mecánica Computacional, vol XXXII, pp. 647-668, 2013,.

[7] O. Poudou, "Modeling and analysis of the dynamics of dry-friction-damped structural systems", Ph.D. thesis, University of Michigan, 2007.

[8] F. Tariku, "Simulation of dynamic mechanical systems with stick-slip friction", Ph.D. thesis, University of New Brunswick, 1998.

[9] Y. Zaspa, Force characteristics of dry friction when contact oscillates. Journal of Friction and Wear, 30(1), pages 17-24, 2009. 